\title{
Information and Communication Technologies (ICTs) Enabling Integrated Primary Care for Complex Patients: A Protocol for a Scoping Review
}

Farah Tahsin ( $\square$ farah.tahsin@hotmail.com )

University of Toronto Dalla Lana School of Public Health https://orcid.org/0000-0001-9077-7670

Alana Armas

: Bridgepoint Collaboratory for Research and Innovation

Apery Kirakalaprathapan

Western University

Heather Cunningham

University of Toronto Gerstein Science Information Centre

Mudathira Kadu

University of Toronto Institute of Health Policy Management and Evaluation

Jasvinei Sritharan

: Bridgepoint Hospital

Carolyn Steele Gray

Bridgepoint Collaboratory for Research and Innovation

\section{Protocol}

Keywords: Patients with complex chronic conditions, integrated primary care, information and communication technologies, primary care, multimorbidity

Posted Date: May 18th, 2021

DOI: https://doi.org/10.21203/rs.3.rs-513310/v1

License: (a) (1) This work is licensed under a Creative Commons Attribution 4.0 International License. Read Full License 


\section{Abstract}

Introduction

An increasing number of individuals are living with multiple chronic conditions, often combined with psychosocial complexities. For these patients with complex conditions, an integrated primary care model that provides care coordination and a team-based approach can help manage their multiple needs. Information and communication technologies (ICTs) are recognized as a critical enabler of integrated primary care. A better understanding of the use of ICTs in an integrated care setting and how ICTs are being leveraged would be beneficial to identify knowledge gaps and could lead to successful implementation for ICT-based interventions.

\section{Objective}

This study will systematically scope the literature on the topic of ICT-enabled integrated healthcare delivery models for patients with complex care needs to identify which technologies have been used in integrated primary care settings.

\section{Method}

This study protocol outlines a scoping review of the peer-reviewed literature, using Arksey and O'Malley's (enhanced by Levac et al.) scoping review methodology. Peer-reviewed- literature will be identified using a multi-database search strategy. The results of the search will be screened, abstracted, and charted in duplicate by 6 research team members.

\section{Discussion}

The key findings of the study will be thematically mapped to describe the implemented ICTs aimed for complex patients within the integrated primary care model and interactions of the elements (ICT, health model, and targeted patients). This review will be the first step to formally identify how ICT is used to support integrated primary health care models. The results will be disseminated through peer-reviewed publications, conference presentations, and special interest groups.

\section{Background}

Across Canada and globally, there is a growing number of individuals living with one or more chronic conditions, alongside of additional psychosocial challenges and socioeconomic factors (1). This patient population often referred to as patients with complex conditions (hereby referred to as PCC), tends to experience disability, functional decline, poor quality of life, and are among the highest users of the healthcare system (2). This patient group also reports higher levels of dissatisfaction with their care compared with less complex counterparts (3). As health systems globally are experiencing a growth in the population of patients with complex health needs (4), many are seeking to shift from old diseasecentric models, focusing on treating illnesses in isolation, towards person-centered and integrated models 
focusing on treating the whole person $(5,6)$. Recent study findings suggest that being able to access person-centered health and social care from providers with whom they have a positive and respectful relationship can improve multimorbid patients' care experience (7).

Primary health care is a crucial starting point in this movement towards whole-person care as it is often patients' first contact of care in their community and where they receive continuous, comprehensive care (8). In the last decade, Wagner's Chronic Care Model (CCM) (9) has been widely used to design coordinated primary health care to resolve the issue of fragmented care when managing chronic conditions. CCM aims to promote care for individuals with chronic conditions that encompass community and health care services. With the recent expansion in the adoption of eHealth technologies to support care delivery, an eHealth enhanced CCM model (eCCM) has been proposed (10). This model acknowledges the importance of information and communication technologies (ICTs) in enabling communication between patients and care providers as part of chronic care management. By using ICTs patients are empowered with information and their care team is better engaged and proactive, which leads to more productive interactions between patients and their primary care teams (10). The CCM and eCCM are useful for helping understand integration in support of chronic disease management for individual patients. However, these individual-level interactions are often couched within a larger system context. Valentijn et al.'s Rainbow model of Integrated Care (RMIC) (11) helps to understand the wider context of integrated care, taking a population-health perspective to identify how health and social care can be integrated at clinical, professional, organizational and system levels. While the emphasis of the CCM is on the provider and patient, the RMIC acknowledges other key players in the delivery of integrated care such as, system and policy-level stakeholders in roles that include governance, finance, and other decision-makers linked to health systems. RMIC considers ICTs as valuable in improving the comprehensiveness of care and communication within and outside of health systems to deliver personcentered care and improve care continuity for patients with complex conditions.

While there have been some case-based studies of the use of ICT in the model of integrated primary health care $(12,13)$, there are currently no reviews available to document what ICTs are being put in place to support these models, and how these ICTs are enabling the process of care delivery. This scoping review seeks to bring together the current research in this space to provide a more extensive overview of the state of ICT in support of integrated primary health care delivery for patients with complex care needs. By scoping the literature we hope to identify promising approaches and current gaps that could help to guide future research, technology development, and implementation in this space.

\section{Study objective}

The objective of this scoping review is to systematically scope the literature on the topic of ICT-enabled integrated primary care delivery models for PCC in order to 1) map how technology is used to support PCC in integrated primary care models and 2) identify specific characteristics and features of ICTs that enable key activities of integrated care delivery. 


\section{Methods}

For this scoping review, we will follow Arksey and O'Malley's (14) scoping review framework that was enhanced by Levac et al. enhanced (15) (Table 1). Each of these stages is described in detail below.

\section{Table 1. Scoping review stages}

1. Identifying the research question

2. Identifying relevant literature

3. Study selection

4. Charting the data

5. Collating, summarizing and reporting findings

6. Consulting and translating knowledge

\section{Stage 1: Identifying the research question}

The primary research question for this study is: "What are the information communication technologies that are used in the delivery of integrated primary care for patients with complex care needs?" The study will also address a secondary research question: "How are these technologies used as part of the model of care?"

To answer the research questions the definitions outlined in Table 2 below were used to guide the development of the major concepts employed in the search strategy.

Table 2: Definitions of eHealth, integrated care, patients with complex chronic needs primary health care 


\begin{tabular}{|ll|}
\hline Concept & Definition \\
$\begin{array}{l}\text { eHealth } \\
(16,17)\end{array}$ & $\begin{array}{l}\text { Health services and information delivered by the combined use of the internet and other } \\
\text { electronic communication technologies. This is a broad definition of eHealth. Thus, } \\
\text { eCCM model is used to categorize eHealth based on the primary functions of a } \\
\text { technology (i.e. decision support). }\end{array}$ \\
\hline $\begin{array}{l}\text { Integrated } \\
\text { care } \\
\text { model } \\
(11)\end{array}$ & $\begin{array}{l}\text { A coordinated collaborative, multidisciplinary (two or more health professionals } \\
\text { involved) and person-centered care delivery system. In this review, an integrated care } \\
\text { model is defined according to Valentijn's Rainbow Model of Integrated Care because of } \\
\text { the model's broader focus on integrated care. }\end{array}$ \\
\hline $\begin{array}{l}\text { Patients } \\
\text { with } \\
\text { complex } \\
\text { care } \\
\text { needs (1) }\end{array}$ & $\begin{array}{l}\text { Individuals with multiple chronic conditions, often aggravated by additional } \\
\text { psychosocial challenges. The complexity of their conditions impacts treatment, health } \\
\text { outcomes, and quality of life. }\end{array}$ \\
\hline $\begin{array}{l}\text { Primary } \\
\text { health } \\
\text { care (18) }\end{array}$ & $\begin{array}{l}\text { The first point of contact that includes key elements such as "disease prevention, health } \\
\text { promotion, population health, and community development within a holistic framework, } \\
\text { to provide essential community-focused health care" (p. 1). }\end{array}$ \\
\hline
\end{tabular}

\section{Stage 2: Identifying relevant literature}

Relevant studies for this review will be identified through searching electronic databases of the published literature which will include: Ovid MEDLINE, Ovid EMBASE, EBSCO CINAHL, Ovid PsycINFO, and the Wiley Cochrane Library. The search strategy for Ovid MEDLINE was first developed with support from the team's information scientist $(\mathrm{HC})$ and has been peer-reviewed using the Peer Review of Electronic Search Strategies (PRESS) tool. The MEDLINE search strategy is included in Appendix A. This search strategy will be then translated over to the remaining databases. A "primary care" filter, which was developed and validated by Gill et al. (19) will be applied to the searches. The literature search will be limited to articles published after 2000; articles published before this date may be less relevant to current ICT integrated primary care model landscape (20).

\section{Stage 3: Study selection}

The study selection process will comprise two levels of screening: (1) title and abstract review and (2) a full-text review. Both levels will be conducted in duplicate using the knowledge synthesis software Covidence. At both levels of screening, any discrepancies between reviewers will be discussed and resolved by the research team collaboratively. The PRISMA framework (21) will be used to guide study selection process (Figure 1) and the Preferred Reporting Items for Systematics Review and Meta-Analysis protocols (PRISMA-P) extension for scoping review guidelines (22) (Appendix 2,Table 1) will be used to guide reporting of the study findings.

To be included the studies will meet the inclusion and exclusion criteria listed in Table 3 . As this scoping review targets general complex patients, we excluded cancer patients and patients with a mental disorder. Patients with cancer often require a different treatment pathway than non-cancer patients (23). Similarly, patients with a mental disorder often receive a different treatment protocol and diagnostic procedure than 
their counterparts with biophysical conditions (24) (25). Studies focused exclusively on individuals younger than 18 are also excluded as ICTs designed for these individuals involve parental consent and subsequently a unique approach to ICT adoption.

\section{Table 3: Inclusion/Exclusion Criteria}

Inclusion Criteria

1. Any published articles including quantitative, qualitative, mixed or multi-methods research, including both comparative (eg. randomized, controlled, cohort, quasi-experimental) and non-comparative (eg. survey, narrative audit) methods, educational materials and reports

2. The intervention has a digital-enabled healthcare model or digital health technology

\section{Exclusion Criteria}

1. The target population for the study is individuals younger than 18 .

2. The target population for the study is individuals with cancer or mental disorder.

3. The intervention is based on an integrated healthcare model or teambased care

4. The intervention includes patients with complex care needs

5. The intervention takes place within primary care.

\section{Stage 4: Data extraction}

All included studies will be reviewed and charted independently by six team members using a pilot-tested data abstraction form. Charting is a technique for organizing and interpreting data by sifting, categorizing, and sorting material, according to key issues and themes (14). We will run the data abstraction pilot-test on a random selection of included articles to ensure consistency across reviewers. Necessary changes will be made and shared with the team before abstracting the remaining articles. The collected data will be stored and compiled in Microsoft Excel for data validation and coding. Table 4 shows the data that will be extracted from the selected literature:

\section{Table 4: Data extraction framework}




\begin{tabular}{|c|c|}
\hline Domain & Information to be extracted \\
\hline \multirow[t]{5}{*}{ Citation summary } & - $\quad$ Author(s) \\
\hline & - Title \\
\hline & - Study citation \\
\hline & - Research design \\
\hline & - Study location \\
\hline \multirow{4}{*}{$\begin{array}{l}\text { Characteristics of the } \\
\text { patient population }\end{array}$} & - Age \\
\hline & - Gender and/or sex \\
\hline & - Type and number of health conditions \\
\hline & $\begin{array}{l}\text { Any additional characteristics provided (ie. marital status, education } \\
\text { level, ethnicity, etc.) }\end{array}$ \\
\hline \multirow[t]{3}{*}{$\begin{array}{l}\text { Characteristics of the } \\
\text { integrated primary care }\end{array}$} & $\begin{array}{l}\text { care) } \\
\text { Setting (inpatient, outpatient, community-based or rehabilitative }\end{array}$ \\
\hline & - Type of service providers and description of roles \\
\hline & - Components of integrated/collaborative care \\
\hline \multirow{3}{*}{$\begin{array}{l}\text { Characteristics of the } \\
\text { implemented } \\
\text { technology }\end{array}$} & $\begin{array}{l}\text { Attributes of technologies (functionality, use, and roles of } \\
\text { technologies in the given care setting) }\end{array}$ \\
\hline & - Aims/purpose of the implemented technology \\
\hline & - Duration of the technological intervention \\
\hline $\begin{array}{l}\text { Facilitators and } \\
\text { barriers to } \\
\text { implementation }\end{array}$ & $\begin{array}{l}\text { Description of the factors that inhibit or facilitate the } \\
\text { implementation of ICTs for complex patients in the integrated primary care } \\
\text { model }\end{array}$ \\
\hline
\end{tabular}

\section{Stage 5: Data summary and synthesis of findings}

The charted data will be organized, coded, and thematically analyzed. Based on the extracted data, each study will be coded based on 1) their primary function (i.e., delivery care tool, self-management support) and 2) the characteristics of integrated care settings (i.e. professional-level integration, clinical level integration). Using a deductive coding approach (26) the key elements of eCCM (10) will be used to determine the primary function of the identified ICTs, whereas RMIC (11) will be used to identify characteristics of the care setting (see Table 5).

After coding of charted data, an evidence map (27) will be generated to help identify research gaps and future research needs in the ICT-field implemented in integrated primary care for patients with complex conditions (Appendix 3,Figure 2). Typically, an evidence map provides a broad overview of existing evidence in a research area and presents results in a user-friendly format (27). This mapping exercise will 
be useful for this scoping review to visually present the comparisons across the interventions, the linkages of evidence, the presence of any contradictory evidence, and potential outlying factors.

Table 5: Key elements of the Rainbow integrated Model of Care (RMIC) and the eHealth enhanced chronic care model (eCCM) 


\section{Concept Key Elements}

RMIC

(11)
Clinical integration: Refers to the coordination of person-focused care in a single process across time, place and discipline

\section{Example}

The care model supports seamless individual care planning, individual case management, etc.

Professional integration: Inter-professional partnerships and coordination of services across multiple disciplines
The care model supports multidisciplinary care within and between the organizations (i.e. general physician and community nurse)

Organizational integration: Inter-organizational coordination of services across multiple organization (e.g., contracting strategic alliances, knowledge networks, mergers), including common governance mechanisms to deliver comprehensive services to a defined population

System integration: A horizontal and vertical alignment of rules and policies within a system
The care model is supported by organizational integration such as coordination of services between long term care and primary health care
The care model is within a
Functional integration: Refers to how the key support functions (i.e., financial, back office and Information management system) are coordinated structured integrated care model network such as the Veterans Health Administration (28)

The care model is situated in an integrated network where financial, information and management system are coordinated (i.e. federal funding allotted in the network)

Normative integration: Refers to the maintenance of coherent and shared mission, vision, and culture between disciplines, external stakeholders, and organizations.

The care model is placed in an integrated network where a mutual aim/vision is shared across stakeholders (i.e. reduce wait time)

Health-related social networks and virtual communities that facilitate care connections

Web-based health platforms, mobile health that support quality improvement

strategies in places to support patient engagements and self-management support

Delivery system design enhancements: Refers to the systems in place to promote teamwork practice to deliver care efficiently

Self-management support enhancements: Refers to the patients' active role in managing their care
Electronic Health Records (EHRs), web-based health platforms that facilitate information sharing

Health apps and online resources that support patients' self-management skills
Clinical decision support enhancements: Refers to providers and health consumers' access to evidence-
Online platforms, EHRs to virtually access protocols and 
based clinical guidelines, protocols, care standards and self-management resources to make an informed decision

Clinical Information Systems Enhancements: Refers to managing information systems (i.e. patient databases, patient portals/Personal health records) to facilitate efficient care guidelines

mHealth apps and online platform to coordinate care and monitor patients' health status

\section{Stage 6: Knowledge translation and stakeholder consultation}

Levac et al. propose that the knowledge translation and consultation phase employ an opportunity for stakeholder engagement (15). To address stakeholder involvement, we will share our preliminary research findings with external healthcare policymakers and researchers to validate search results and support knowledge translation efforts. Special interest group members of the International Foundation for Integrated Care (IFIC) will be consulted for expert opinions on our scoping review findings (29). This special interest group consists of a wide range of membership with representation from research and academia, health care management, front line providers, government authorities/policymakers, patients and families, as well as private stakeholders. Members of this special interest group have a background in the development, adoption, and/or evaluation of digital health solutions in models of integrated care. Furthermore, we will prepare educational materials and presentations to disseminate study findings to multidisciplinary health teams, caregivers, and patients and at relevant national and international conferences. Results will also be published in a peer-reviewed journal and infographics will be developed for easy uptake by a wider user audience.

\section{Discussion}

To our knowledge, this is the first review that will comprehensively identify the existing ICTs used to support the health and social care needs of complex patients within an integrated primary care model. This scoping review will inform future research and key stakeholders about the important roles that these technologies play to improve patients' and providers' care experience. In addition, this review will identify potential gaps in the current ICT landscape that could improve care coordination, communication, and disease management among complex patients.

\section{Abbreviations}

PCC: Patients with complex conditions

ICTs: Information and communication technologies

RMIC: Rainbow model of integrated care

eCCM: eHealth enhanced chronic care model

IFIC: International foundation for integrated care 


\section{Declarations}

Ethics approval and consent to participate: Not Applicable

Consent for publication: Not Applicable.

Availability of data and materials: Search strategies are available in the appendix 1.

Competing interests: Not applicable

Funding: The study did not receive any funding

Author contributions: FT: conceptualization, writing-first draft; AA: conceptualizationwriting-first draft, reviewing and editing; HC: writing-critical review, search strategy development; $\mathbf{A K}$ : writing-first draft, and editing; MK: writing-critical review and editing; JS: writing-critical review and editing; CSG: conceived and designed the study, writing-initial draft, reviewing and editing, supervision of the project.

Guarantor: Farah Tahsin

Acknowledgements: We would like to thank Dr. Heather Cunningham for contributing her time to this study.

\section{References}

1. Schaink AK, Kuluski K, Lyons RF, Fortin M, Jadad AR, Upshur R, et al. A Scoping Review and Thematic Classification of Patient Complexity: Offering a Unifying Framework. Journal of Comorbidity. 2012 Jan;2(1):1-9.

2. Hajat C, Stein E. The global burden of multiple chronic conditions: A narrative revie[1] C. Hajat and E. Stein, "The global burden of multiple chronic conditions: A narrative review," Preventive Medicine Reports, vol. 12. Elsevier Inc., pp. 284-293, 01-Dec-2018.w. Vol. 12, Preventive Medicine Reports. Elsevier Inc.; 2018. p. 284-93.

3. Burgers JS, Voerman GE, Grol R, Faber MJ, Schneider EC. Quality and coordination of care for patients with multiple conditions: results from an international survey of patient experience. Evaluation \& the health professions. 2010 Sep;33(3):343-64.

4. Roberts KC, Rao DP, Bennett TL, Loukine L, Jayaraman GC. Prevalence and patterns of chronic disease multimorbidity and associated determinants in Canada. Health Promotion and Chronic Disease Prevention in Canada. 2015;35(6):87-94.

5. Bickerstaffe S. Towards whole person care. 2013;(December):1-26.

6. Reuben DB, Tinetti ME. Goal-oriented patient care - An alternative health outcomes paradigm. Vol. 366, New England Journal of Medicine. Massachussetts Medical Society; 2012. p. 777-9.

7. Kuluski K, Peckham A, Gill A, Gagnon D, Wong-Cornall CW, McKillop A, et al. What is Important to Older People with Multimorbidity and Their Caregivers? Identifying Attributes of Person Centered 
Care from the User Perspective. International Journal of Integrated Care. 2019;19(3).

8. Starfield B. Primary care: An increasingly important contributor to effectiveness, equity, and efficiency of health services. SESPAS report 2012. Gaceta Sanitaria. 2012;26(SUPPL.1):20-6.

9. Wagner EH. Chronic disease management: what will it take to improve care for chronic illness? Effective clinical practice: ECP. 1(1):2-4.

10. Gee PM, Greenwood DA, Paterniti DA, Ward D, Miller LMS. The eHealth enhanced chronic care model: A theory derivation approach. Journal of Medical Internet Research. 2015 Apr 1;17(4):e86.

11. Valentijn PP, Schepman SM, Opheij W, Bruijnzeels MA. Understanding integrated care: A comprehensive conceptual framework based on the integrative functions of primary care. International Journal of Integrated Care. 2013;13(JANUARY-MARCH 2).

12. Morton K, Dennison L, May C, Murray E, Little P, Mcmanus RJ, et al. Using digital interventions for self-management of chronic physical health conditions: A meta-ethnography review of published studies Europe PMC Funders Group. Patient Educ Couns. 2017;100(4):616-35.

13. Steele Gray C, Barnsley J, Gagnon D, Belzile L, Kenealy T, Shaw J, et al. Using information communication technology in models of integrated community-based primary health care: Learning from the iCOACH case studies. Implementation Science. 2018 Dec 26;13(1):1-14.

14. Arksey H, O’Malley L. Scoping studies: Towards a methodological framework. International Journal of Social Research Methodology: Theory and Practice. 2005 Feb;8(1):19-32.

15. Levac D, Colquhoun H, O’Brien KK. Scoping studies: Advancing the methodology. Implementation Science. 2010 Sep 20;5(1).

16. Eysenbach G. What is e-health? Journal of Medical Internet Research. 2001;3(2):1-5.

17. Shaw T, McGregor D, Brunner M, Keep M, Janssen A, Barnet S. What is eHealth (6)? Development of a Conceptual Model for eHealth: Qualitative Study with Key Informants. Journal of medical Internet research. 2017;19(10):e324.

18. Thomas-Maclean R, Tarlier D, Ackroyd-Stolarz S, Fortin M, Stewart M. TUTOR-PHC 2003/2004 RESEARCH TRAINEES “NO COOKIE-CUTTER RESPONSE” CONCEPTUALIZING PRIMARY HEALTH CARE 1.

19. Gill PJ, Roberts NW, Wang KY, Heneghan C. Development of a search filter for identifying studies completed in primary care. Family Practice. 2014;31(6):739-45.

20. Everall AC, Guilcher SJT, Cadel L, Asif M, Li J, Kuluski | Kerry, et al. Patient and caregiver experience with delayed discharge from a hospital setting: A scoping review. Health Expectations. 2019;22:86373.

21. Moher D, Hopewell S, Schulz KF, Montori V, Gøtzsche PC, Devereaux PJ, et al. CONSORT 2010 Explanation and Elaboration: updated guidelines for reporting parallel group randomised trials. Journal of Clinical Epidemiology [Internet]. 2010;63(8):e1-37. Available from: http://dx.doi.org/10.1016/j.jclinepi.2010.03.004 
22. Liberati A, Altman DG, Tetzlaff J, Mulrow C, Gøtzsche PC, loannidis JPA, et al. The PRISMA statement for reporting systematic reviews and meta-analyses of studies that evaluate healthcare interventions: explanation and elaboration. BMJ (Clinical research ed) [Internet]. 2009 Jul 21 [cited 2021 May 10];339. Available from: http://www.bmj.com/

23. Evans JM, Matheson G, Buchman S, MacKinnon M, Meertens E, Ross J, et al. Integrating cancer care beyond the hospital and across the cancer pathway: a patient-centred approach. Healthcare quarterly (Toronto, Ont). 2015;17:28-32.

24. Nutting PA, Gallagher KM, Riley K, White S, Dietrich AJ, Dickinson WP. Implementing a depression improvement intervention in five health care organizations: Experience from the RESPECT-Depression trial. Administration and Policy in Mental Health and Mental Health Services Research. 2007 Mar 13;34(2):127-37.

25. Thielke S, Vannoy S, Unützer J. Integrating Mental Health and Primary Care. Vol. 34, Primary Care Clinics in Office Practice. 2007. p. 571-92.

26. Creswell JW. Qualitative Inquiry \& Research Design. Sage Publications, Inc. 2007.

27. Miake-Lye IM, Hempel S, Shanman R, Shekelle PG. What is an evidence map? A systematic review of published evidence maps and their definitions, methods, and products. Systematic Reviews. 2016 Feb 10;5(1):28.

28. Perlin JB, Kolodner RM, Roswell RH. The Veterans Health Administration: quality, value, accountability, and information as transforming strategies for patient-centered care. HealthcarePapers. 2005;5(4):10-24.

29. The International Foundation for Integrated Care IFIC [Internet]. [cited 2020 Apr 18]. Available from: https://integratedcarefoundation.org/

\section{Figures}




\section{PRISMA 2009 Flow Diagram}
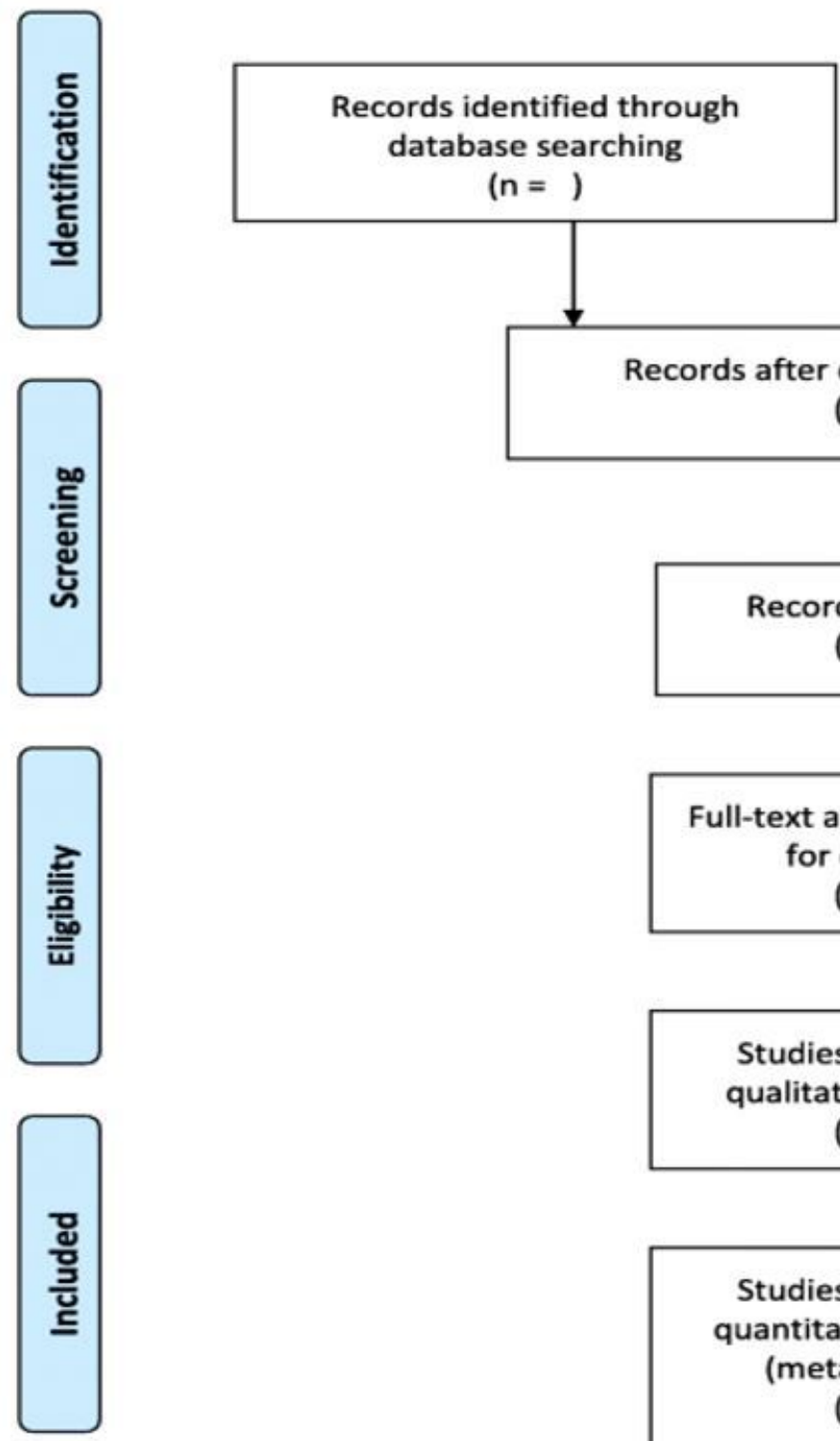

Additional records identified through other sources $(n=)$ $(n=)$

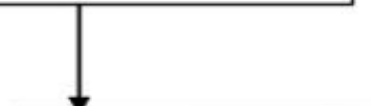

Records after duplicates removed $(n=)$

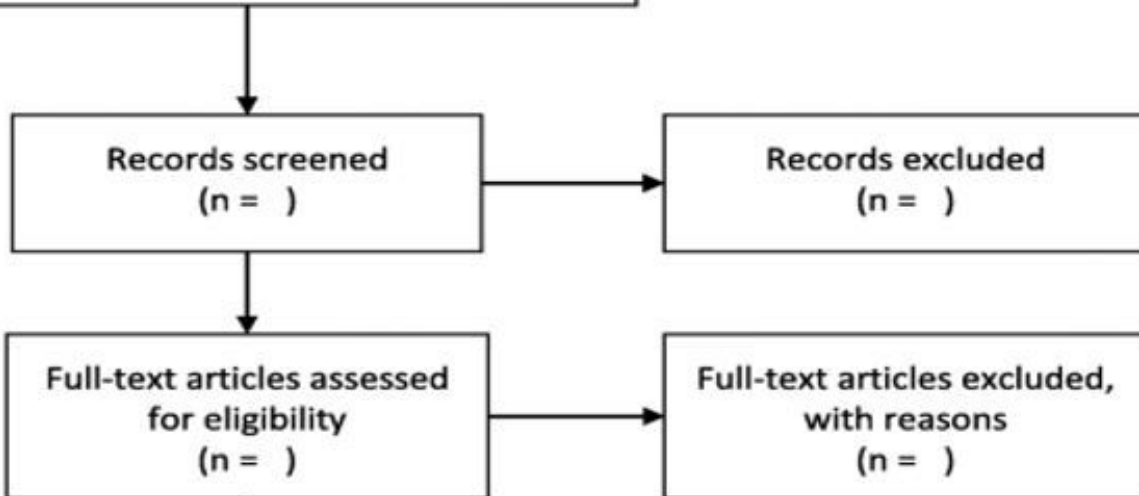

Studies included in qualitative synthesis $(n=)$

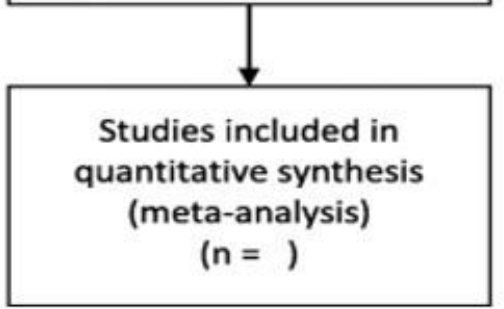

\section{Figure 1}

Prisma 200 Flow diagram (21)

\section{Supplementary Files}

This is a list of supplementary files associated with this preprint. Click to download.

- Appendix2Table1PRISMAScR.pdf

- Appendix1Searchstrategy.pdf 